\title{
Population status of the Alaotran gentle lemur Hapalemur griseus alaotrensis
}

\author{
Thomas Mutschler, A. Jeannicq Randrianarisoa and Anna T. C. Feistner
}

\begin{abstract}
The results of a field census of Alaotran gentle lemurs Hapalemur griseus alaotrensis carried out in February and March 1999 are reported. The results are compared with a census carried out 5 years earlier in 1994. Both censuses followed the same methods and were carried out by the same team, using direct observation from canoe. Mean group encounter rates for the lemur were assessed in each location, which allowed us to calculate the relative group density in each site. Qualitative information on habitat condition (on the basis of plant diversity, vegetation height and evidence of burning) was gathered at each site. Additional information, mainly on lemur hunting, was acquired through interviews with local people. In most sites
\end{abstract}

\section{Introduction}

The papyrus and reed beds of Madagascar's biggest lake, Lac Alaotra, harbour a cryptic lemur, the Alaotran gentle lemur Hapalemur griseus alaotrensis, locally called bandro. Its occurrence is strictly limited to the Alaotran wetland, no part of which is legally protected. In the last decade it has become clear that the Alaotran gentle lemur is one of the more threatened extant lemurs (Mittermeier et al., 1992, 1994) and that its conservation status should be monitored closely. The Durrell Wildlife Conservation Trust, which has been working for the conservation of the Alaotran gentle lemur on several fronts since 1990 (reviewed in Feistner, 1999), undertook a survey of its distribution and population status in 1994. Evidence was provided that the lemur's distribution area had shrunk in recent times to some 20,000 ha and resulted in an estimation of a total population size of 7500-10,700 individuals, with the lower figure likely to be the more realistic (Mutschler \& Feistner, 1995). The lemurs occurred in two subpopulations, a very small one in the northern part of the lake (around Belempona peninsula) and a large one in the southern and western parts of the marshland (south of Anony confluence).

Anna T. C. Feistner (corresponding author) Durrell Wildlife Conservation Trust, Les Augrès Manor, Trinity, Jersey JE3 5BP, Channel Islands, British Isles. E-mail: afeistner@durrell.org

Thomas Mutschler and A. Jeannicq Randrianarisoa Durrell Wildlife Conservation Trust, BP 8511, Antananarivo 101, Madagascar

Revised manuscript accepted for publication 16 November 2000 group encounter rate was at least 50 per cent lower than 5 years before. Taking into account unusually low water levels because of a drought at Lac Alaotra in 1999, we estimate that these encounter rates reflect a 30 per cent decline in the total population size over the last 5 years. The main cause of this dramatic decrease was humaninduced fire (habitat degradation) coupled with heavy hunting (poaching of lemurs for food). The importance of assessing regularly both population status and threats, and of adjusting conservation actions accordingly is emphasized.

Keywords Alaotra, conservation, hunting, lemurs, Madagascar.

The survey in 1994 showed that the continued survival of this marsh-dwelling lemur was threatened by habitat destruction (marshes transformed into agricultural areas) and by uncontrolled marsh fires. Moreover, the lemur was also reported to suffer considerably from poaching (Feistner \& Rakotoarinosy, 1993; Mutschler \& Feistner, 1995; Pidgeon, 1996).

Following the 1994 survey, several actions and studies towards the in-situ and $e x$-situ protection of the lemur and towards the conservation of the Alaotran wetlands were initiated (e.g. Feistner \& Beattie, 1998; Durbin, 1999; Feistner, 1999; Mutschler, 1999a, b; Mutschler et al., 2000). Five years after the first census, a second survey was undertaken to re-assess the population and conservation status of the lemur. The results of the 1999 survey are reported here and compared with the situation in 1994 (Mutschler \& Feistner, 1995).

\section{Methods}

The Alaotran wetland was surveyed for 2 months (February and March) in 1999. The aim was to determine population densities of the Alaotran gentle lemur and assess the condition of marsh vegetation. The Alaotran gentle lemur and its habitat were surveyed at seven different sites around Lac Alaotra: Belempona, Vohimarina, Vohitsara, Anororo, Andilana Atsimo, Ambodivoara and Andreba (Fig. 1). Methods strictly followed the 1994 survey (Mutschler et al., 1994; Mutschler \& Feistner, 1995). Animals were searched for 
round the lake edge or from existing channels (cut by fishermen) in the vegetation. All observations were carried out by the same team and during the same periods of the day (05.00-10.00 and 15.30-18.30 hrs). When lemur groups were encountered, they were observed for a maximum of $15 \mathrm{~min}$ and then the search continued. The survey resulted in a mean encounter rate for each location (groups encountered per hour of search) and an assessment of relative group density of the lemurs at that site. Relative group densities for the different sites were compared with each other and to the relative density found in 1994 .

Qualitative information on habitat condition was collected during the animal census and is described for each site. Habitat condition was classified in three categories: (1) Good (vegetation not burnt for at least 5 years): vegetation (papyrus and reed) $3-5 \mathrm{~m}$ high; dense, diverse undergrowth up to about $1 \mathrm{~m}$ in height; papyrus or reeds covered with dense creepers (See Plate 1(a)). (2) Medium (vegetation recovering from fire; not burnt for at least 2 years): dense, uniformly sized papyrus vegetation up to $3 \mathrm{~m}$ in height; undergrowth mainly composed of fern; no or only very few creepers. (3) Poor (areas burnt within the last 2 years; cleared areas): very dense, uniform grass and papyrus vegeta-

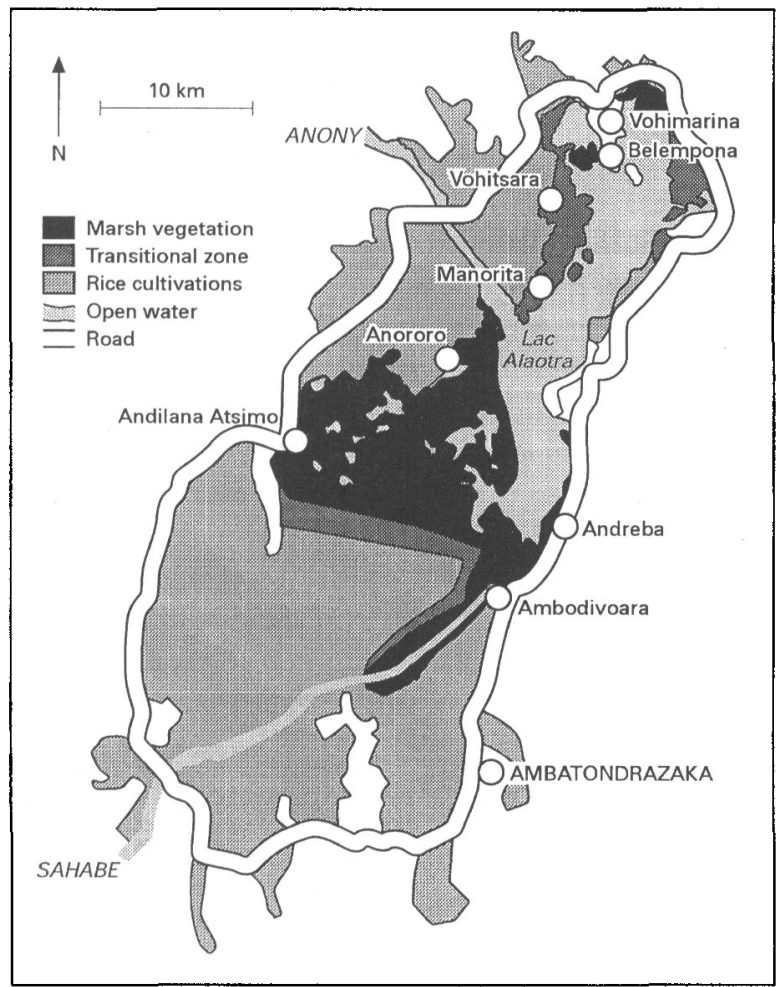

Fig. 1 Map of the Alaotran wetland (modified after Mutschler \& Feistner, 1995). tion up to $1 \mathrm{~m}$; little undergrowth; no creepers. Other information presented in this paper results from ad libitum observations during lemur censuses and/or from interviews with local people. Climatic data are from the Meteorological Station Ambohitsilaozana, near Ambodivoara.

\section{Results}

In the first 2 months of 1999 very little rain fell (Fig. 2), resulting in both an unusual drought in the Alaotran basin (at a time which is usually the wet season) and in a markedly lower water level in the lake than in early 1994.

In the northern parts of the lake (north of the Anony confluence) most marsh areas had long disappeared and are now cultivated or used for grazing cattle. The remaining papyrus marshes cover only a small surface area, are heavily fragmented and are mostly of poor to medium quality. Compared with 1994, the situation has not changed much. The marsh surface in the area between Manorita and Vohitsara seemed to be slightly reduced, and around the Belempona peninsula it remained unchanged. On the eastern side of this peninsula, however, we found the remaining marsh areas in a slightly better condition (smaller burnt area) than 5 years ago. A further positive trend was found in Vohitsara, where about 5 ha of papyrus marsh had been successfully replanted. The initiator of the planting aims to repeat this action on an annual basis. Overall, marsh surface and habitat quality had not significantly changed in the northern part of the lake since 1994.

The northern subpopulation of the Alaotran gentle lemur was estimated to consist of about 60 individuals in 1994. In 1999, no lemurs were encountered during

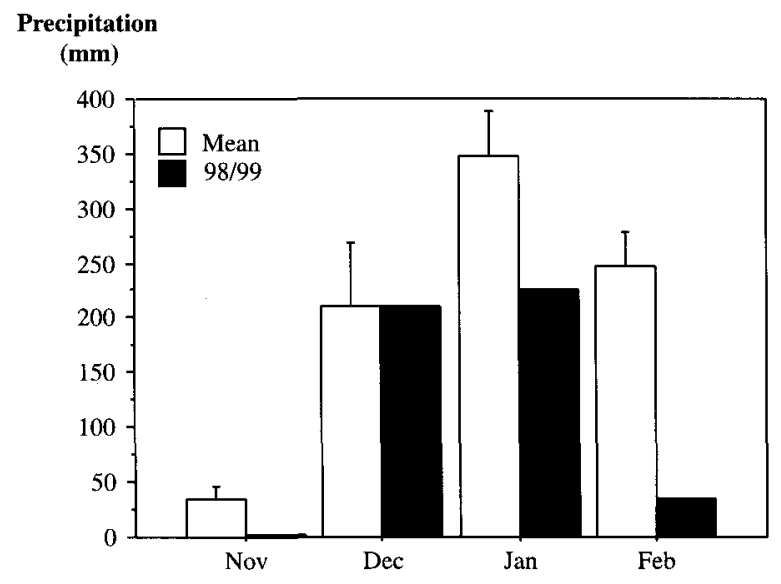

Fig. 2 Precipitation from November 1998 to February 1999, compared with the corresponding mean values $( \pm$ SEM) over the last 9 years (January 1990-October 1998). 

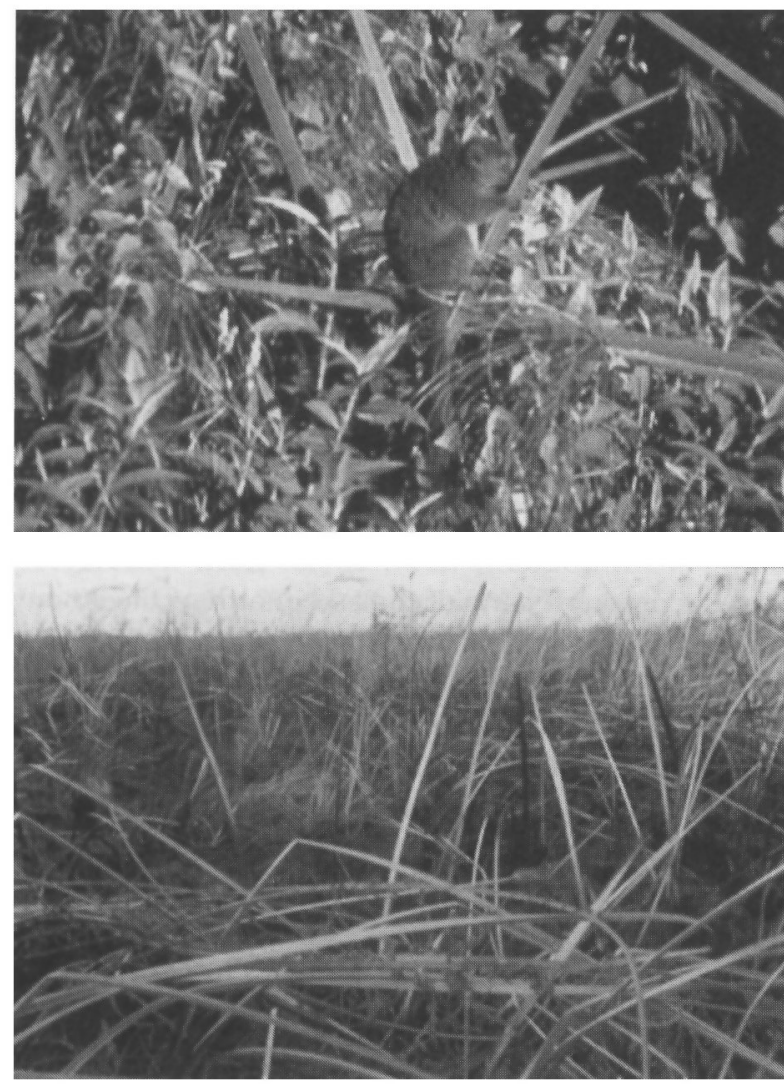

Plate 1 (a) Wild Alaotran gentle lemur in good habitat: dense papyrus and reed vegetation $3-5 \mathrm{~m}$ high covered with dense creepers, and a diverse dense undergrowth (Thomas Mutschler); (b) the same habitat after human-set fires (Anna Feistner).

more than $55 \mathrm{~h}$ of survey in the north of Lac Alaotra (Table 1). The only mammals seen in the northern marshes were several black rats Rattus rattus. The only evidence for the continued persistence of Alaotran gentle lemurs in the north was a few vocalizations.

Around Anororo some marsh areas north of the village had been transformed into cultivated land (rice fields) and pasture. Overall, the marsh surface around

Table 1 Survey results for Alaotran gentle lemurs in 1999.

\begin{tabular}{llll}
\hline Site & $\begin{array}{l}\text { Census } \\
\text { time } \\
\text { (min) }\end{array}$ & $\begin{array}{l}\text { Number of } \\
\text { groups } \\
\text { (individuals) }\end{array}$ & $\begin{array}{l}\text { Groups } \\
\text { encountered } \\
\text { per hour }\end{array}$ \\
\hline $\begin{array}{l}\text { North } \\
\quad \text { Belempona }\end{array}$ & 1295 & $0(0)$ & - \\
$\quad \begin{array}{l}\text { Vohimarina } \\
\quad \text { Vohitsara }\end{array}$ & 1380 & $0(0)$ & - \\
Anororo & 675 & $0(0)$ & - \\
Andilana-Sud & 1650 & $7(20)$ & 0.255 \\
Andreba & 1780 & $2(8)$ & 0.067 \\
Ambodivoara & 1670 & $10(23)$ & 0.359 \\
\hline
\end{tabular}

the village was still about the same as in 1994, but the marshes had clearly suffered from large-scale burning during the last five years (see Plate 1(b)), resulting in largely degraded marshes of poor to medium quality, with small and uniform vegetation.

The relative density of the Alaotran gentle lemur around Anororo was markedly lower (about 50 per cent) than that found in 1994 (Table 2). A negative relationship between proximity to the village and lemur density was also apparent. Near the village, marshes were almost depleted of the lemur, whereas more remote areas still contained some groups. Despite a local fady (traditional taboo) forbidding people to carry hunted lemurs into the village of Anororo, diverse sources reported to us that the lemur was now heavily poached in Anororo. Several independent sources estimated that in 1998 more than 500 dead lemurs were brought into Anororo.

East of Andilana Atsimo the marshland is vast, and contains several lakes and pools. This area was considered as one of the last strongholds of the Alaotran gentle lemur in 1994. The present study found the marshes to be just as extensive, but severely degraded. All accessible areas up to $6 \mathrm{~km}$ from the village had been burnt at least once within the last five years. The marsh vegetation around Andilana Atsimo was largely deprived of cover and food for the Aloatran gentle lemur and most papyrus was so small that it could hardly support the weight of an adult lemur.

The relative density of lemurs had dropped to near zero in Andilana Atsimo - only two groups were encountered during $30 \mathrm{~h}$ of survey (Table 1). Although we worked during daylight hours only, ironically we spotted more nocturnal mouse lemurs Microcebus rufus than cathemeral gentle lemurs during the survey in Andilana Atsimo. Reports of heavy poaching of the Alaotran gentle lemur in recent years may explain this alarming drop in encounter rate, with close to a thousand lemurs estimated killed at Andilana Atsimo during 1998.

The marshes around Andreba had not changed in surface area over the last five years. Most vegetation was tall, diverse and structured, providing both cover and

Table 2 Encounter rates of Alaotran gentle lemur at the survey sites in 1994 and 1999.

\begin{tabular}{lll}
\hline & \multicolumn{2}{l}{ Groups encountered per hour of survey } \\
\cline { 3 - 3 } Site & 1994 & 1999 \\
\hline North Alaotra & 0.224 & 0.000 \\
Anororo & 0.513 & 0.255 \\
Andilana Atsimo & 0.471 & 0.067 \\
Andreba & 0.716 & 0.359 \\
Ambodivoara & Not surveyed & 0.404 \\
\hline
\end{tabular}


food for the Alaotran gentle lemur. There were several burnt patches, although no more than in 1994, but the marsh was increasingly degraded by fire towards the south-west.

As in Anororo, the encounter rate in the Andreba marshes in 1999 was 50 per cent of that in 1994 (Table 2), but it was obvious that encounter rates were different for the northern and the southern part of the Andreba marshes (Table 3). North of Andreba we encountered lemurs with a frequency of 0.588 groups per hour of search, whereas only 0.188 groups per hour were encountered in the south. This difference, however, was not reflected in the habitat quality of the two sites, which appeared similar. It seems that the long-term presence of researchers in Andreba (the researchers live in the village and work closely with the local people) and the coloured collars of the study animals are the reason for the difference. The villagers link the study animals with the researchers and so the lemurs are regarded as the 'property' of the scientists. People from other areas of the lake also seem hesitant about capturing collared animals round Andreba. In a marsh area about $2 \mathrm{~km}$ long and $200 \mathrm{~m}$ wide to the north of Andreba, the total population of lemurs has been monitored regularly since early 1996, and all adult animals in this site have been marked with coloured collars. In February 1996 the population in this site was 35 animals, whereas 3 years later, in February 1999, the population had increased to 55 (Nievergelt, 1999). Thus, the population size of the marked animals had increased substantially, in contrast to all other surveyed populations. Moreover, people from Andreba seemed to be more aware about the protected status of the lemurs and only poached them rarely. Nevertheless, the Alaotran gentle lemur does suffer some losses in the Andreba area, because the southern region of Andreba is also used by the fishermen of Ambodivoara, who are known as the most skilled lemur hunters of Lac Alaotra.

In the area surrounding Ambodivoara, the Sahabe river and a canal system leading to the open lake were

Table 3 Alaotran gentle lemur survey 1999; data for Andreba and Ambodivoara split into different regions.

\begin{tabular}{llll}
\hline & $\begin{array}{l}\text { Census } \\
\text { time } \\
\text { (min) }\end{array}$ & $\begin{array}{l}\text { Number of } \\
\text { groups } \\
\text { (individuals) }\end{array}$ & $\begin{array}{l}\text { Groups } \\
\text { encountered } \\
\text { per hour }\end{array}$ \\
\hline Andreba & & & \\
$\quad \begin{array}{l}\text { North } \\
\text { South \& west }\end{array}$ & 715 & $7(16)$ & 0.588 \\
Ambodivoara & 955 & $3(7)$ & 0.188 \\
$\quad$ & & $10(28)$ & 0.663 \\
Sahabe & 905 & $2(4)$ & 0.137 \\
\hline
\end{tabular}

surveyed. This area was not visited in 1994. The conditions of the marshes along the Sahabe and along the canal system were very different, so the two areas are considered separately. On the edge of the Sahabe, the marsh vegetation was the most undisturbed we saw during the 1999 survey. Most reed and papyrus beds were 4-5 $\mathrm{m}$ high, had a dense under-storey and were covered with creepers, thus providing plenty of food and cover for the lemurs. Nevertheless, even along the Sahabe, some small areas were burnt, but overall these marshes were the best quality habitat for Alaotran gentle lemurs. By contrast, along the canal system leading from Ambodivoara to Lac Alaotra (Canal Rabenony, Canal Andriatsimiavona, Canal Vohitraivo, Canal Mahakary), the marshes were severely degraded, and all had been burnt within the last three years. Most of the regenerating marshes were covered only with small and uniform papyrus plants and a few grasses. Other areas had even been cleared after burning and, therefore, no longer contained any papyrus vegetation. Moreover, several small rice fields were seen within this marsh area. The condition of the marshes along these canals was comparable with that of the marshes of Andilana Atsimo or even worse (i.e. converted to rice plantations).

The overall encounter rate for lemurs around Ambodivoara (including data from both the Sahabe and the canal system) was higher than all other sites visited during the 1999 survey (Table 1). The dichotomy in habitat quality between the Sahabe and the canal system was also found in the relative density of lemurs (Table 3). Along the Sahabe, lemur groups were encountered at a frequency of 0.663 per hour, almost equalling the highest relative density found during the survey in 1994 (0.716 in Andreba; Table 2), whereas only 0.137 groups per hour were encountered along the canal system (Table 2).

Fishermen from Ambodivoara are known locally to be skilled hunters of the Alaotran gentle lemur. The lemurs are hunted to be kept as pets or are killed and eaten. During our work in and around Ambodivoara we were asked many times whether we would like to purchase live or dead lemurs. Although it was obvious that hunting was frequent, the reports of the number of hunted lemurs varied considerably, so it is difficult to estimate the number of animals poached in Ambodivoara.

\section{Discussion}

The results of the 1999 survey are extremely alarming. In most sites visited around Lac Alaotra in 1999, the group encounter rate of the Alaotran gentle lemur was at least 50 per cent lower than in 1994. Does this marked drop in relative density of the lemurs translate directly into an equal reduction of total population size or does 
it correspond to external differences (e.g. methods, climate) between the two surveys?

The 1999 survey was carried out during February and March, supposed to be the time of highest water level at Lac Alaotra and, thus, the time when marshes are most accessible. The methods strictly followed those of the 1994 survey and both surveys were carried out by the same team. Thus, the study was designed to keep as many factors constant as possible. Nevertheless, there were differences between the two studies. The most noticeable difference was the unusually low water level of Lac Alaotra in early 1999, caused by a severe drought in the Alaotran basin during January and February 1999. This drought influenced the study in two ways: (1) some marsh areas in Anororo and Andreba, which were surveyed in 1994, were not accessible in 1999 and (2) the under-storey of the marshes was flooded for a brief time only and hence stayed very dense. We speculate that these factors most probably resulted in a slightly lower visibility than in 1994.

Lower visibility and reduced marsh accessibility could, of course, result in a lowering of the encounter rate of the Alaotran gentle lemur in the Alaotran wetland, but it seems highly unlikely that they are responsible for the observed drop of 50 per cent or more. On the contrary, the results of the recent survey support the idea that the drop in group encounter rate is mostly caused by a real reduction in the total population size of wild Alaotran gentle lemurs. First, reduced accessibility of the marshes only influenced the results of the surveys in Anororo and Andreba, whereas survey areas in the northern parts of Lac Alaotra and in Andilana Atsimo were as accessible as in 1994. Nevertheless, encounter rates in these sites (Belempona, Vohimarina and Andilana Atsimo) dropped to near zero. Second, along the Sahabe river, where marshes were mostly undisturbed, and north of Andreba, where animals were collared, group encounter rates were within the range of the values found during the 1994 survey. The highest group encounter rates found in 1999 were only 10-20 per cent lower than the highest rate found in 1994, and it seems that this 10-20 per cent reduction is best explained by the effects of low water level. Even if the low water level accounts for 20 per cent of the observed drop in encounter rate however, the result is still shocking: the density of wild Alaotran gentle lemurs has decreased by at least 30 per cent in the last 5 years!

If we look more closely, the picture gets even more worrying. The subpopulation in the north is on the brink of extinction. Vast marsh areas around Andilana Atsimo and along the canals leading from Ambodivoara to Lac Alaotra are almost empty of lemurs, and marsh areas close to Anororo and south of Andreba show tendencies of going the same way in the near future.
What caused this tremendous decline in lemurs? The extent of the marsh surface in all visited sites has not changed much within the last 5 years. Thus habitat destruction cannot be the reason for the reduced density. The condition or quality of the marsh vegetation, however, has changed since 1994. Large areas of marsh have suffered from human-induced fires during the last 5 years. Most of these burnt areas have the potential to recover from fire, but it will take time and the burnt areas will remain degraded for several years (especially if burnt several years in succession). In sites with heavily burnt areas we found the lowest relative densities of lemurs. Low relative densities of Alaotran gentle lemurs, however, are not only proportional to the burnt marsh surfaces but also coincide with reports of heavy poaching.

Rumours about continued poaching of Alaotran gentle lemurs in Andilana Atsimo have been frequent in recent years. During the 1999 study, several sources from the village itself finally confirmed these rumours, and suggested that about 1000 lemurs were hunted in Andilana Atsimo during the dry season of 1998. Another focus for hunting seems to be Ambodivoara. The fishermen from this village own the smallest dugout canoes on the lake, with which they can penetrate deep into the marshes. Furthermore, they are notorious for their hunting skills. And even in Anororo, the local fady, still largely respected in 1994, has obviously lost its power.

Although this study was not designed to evaluate the number of hunted and killed lemurs, the reports on hunting were very detailed and were confirmed from several independent sources. Moreover, the information on the small population of marked animals in the north of Andreba supports the idea that severe poaching is the cause of population reduction. The size of the north Andreba population has increased noticeably, from 35 to 55 animals over the three-year period since the animals were marked with collars as part of a long-term study (Nievergelt et al., 1998; Mutschler, 1999a; Nievergelt, 1999; Mutschler et al., 2000). This implies that the density of Alaotran gentle lemurs around the lake is well below carrying capacity, and that low density is because of heavy hunting pressure. Thus, we should take the reports on poaching at face value. They suggest that $1000-3000$ wild Alaotran gentle lemurs were lost to poachers in 1998.

In 1994, the Alaotran gentle lemur was thought to be mostly threatened by habitat destruction and fragmentation (Mutschler \& Feistner, 1995). Although we then concluded that hunting activity was far from trivial, we did not consider it as a primary threat. Now, however, poaching has become the prime threat to this lemur. Two main factors have reduced the number of wild Alaotran gentle lemurs during the last 5 years: fires and poaching. These two often go hand-in-hand, because fire 
seems to be the most successful way of hunting lemurs (Feistner \& Rakotoarinosy, 1993). It is not clear whether we underestimated the effect of poaching in 1994 or whether poaching has increased significantly over the last 5 years, but we suspect the latter. It is clear that habitat protection alone is not sufficient for the conservation of the Alaotran gentle lemur. Immediate action to protect it against poaching (the lemur is legally protected) is urgently needed; without it the flagship species of the Alaotran wetland will soon be extinct in the wild.

The importance of regular monitoring of the status of animals considered threatened has been emphasized, and this study has also demonstrated the rapidity with which a situation can change, moving an animal from endangered to critical in a short space of time. It also emphasizes the need to regularly adjust conservation actions (for example from habitat restoration to direct species protection) in the light of findings from research aimed at monitoring conservation status.

\section{Acknowledgements}

Fieldwork was carried out under the Accord between the Durrell Wildlife Conservation Trust and the Government of Madagascar through the Tripartite Commission. We thank the governmental institutions of Madagascar for permission to conduct the study.

\section{References}

Durbin, J. (1999) Lemurs as flagships for conservation in Madagascar. In New Directions in Lemur Studies (eds B. Rakotosamimanana, H. Rasamimanana, J. U. Ganzhorn and S. M. Goodman), pp. 269-281. Plenum Press, New York.

Feistner, A.T.C. \& Beattie, J.B. (1998) International Studbook for the Alaotran Gentle Lemur Hapalemur griseus alaotrensis: Number Two 1997. Jersey Wildlife Preservation Trust, Jersey.

Feistner, A.T.C. (1999) Conservation of the Alaotran gentle lemur: a multidisciplinary approach. In New Directions in Lemur Studies (eds B. Rakotosamimanana, H. Rasamimanana, J. U. Ganzhorn and S. M. Goodman), pp. 241-248. Plenum Press, New York.

Feistner, A.T.C. \& Rakotoarinosy, M. (1993) Conservation of gentle lemur Hapalemur griseus alaotrensis at Lac Alaotra Madagascar: local knowledge. Dodo, Journal of the Wildife Preservation Trusts, 29, 54-65.

Mittermeier, R.A., Konstant, W.R., Nicoll, M.E. \& Langrand, O. (1992) Lemurs of Madagascar: an Action Plan for their Conservation 1993-99. IUCN, Gland, Switzerland.

Mittermeier, R.A., Tattersall, I., Konstant, W.R., Meyers, D.M. \& Mast, R.B. (1994) Lemurs of Madagascar. Conservation International, Washington, DC.

Mutschler, T. (1999a) The Alaotran gentle lemur (Hapalemur griseus alaotrensis): a study in behavioural ecology. PhD Thesis, University of Zürich, Zürich.

Mutschler, T. (1999b) Folivory in a small-bodied lemur: the nutrition of the Alaotran gentle lemur (Hapalemur griseus alaotrensis). In New Directions in Lemur Studies (eds B. Rakotosamimanana, H. Rasamimanana, J. U. Ganzhorn and S. M. Goodman), pp. 221-239. Plenum Press, New York.

Mutschler, T. \& Feistner, A.T.C. (1995) Conservation status and distribution of the Alaotran gentle lemur Hapalemur griseus alaotrensis. Oryx, 29, 267-274.

Mutschler, T., Nievergelt, C. \& Feistner, A.T.C. (1994) Biology and Conservation of Hapalemur griseus alaotrensis. Unpublished report. Jersey Wildlife Preservation Trust, Jersey.

Mutschler, T., Nievergelt, C.M. \& Feistner, A.T.C. (2000) The social organization of the Alaotran gentle lemur (Hapalemur griseus alaotrensis). American Journal of Primatology, 50, 9-24.

Nievergelt, C.M. (1999) Genetics of wild-living gentle lemurs. In Primatology and Anthropology into the Third Millenium, Centenary Congress of the Anthropological Institute and Museum in Zürich, p. 38. University of Zürich, Zürich.

Nievergelt, C., Mutschler, T. \& Feistner, A.T.C. (1998) Group encounters and territoriality in wild Alaotran gentle lemurs (Hapalemur griseus alaotrensis). American Journal of Primatology, 46, 251-258.

Pidgeon, M. (1996) An Ecological Survey of Lake Alaotra and Wetlands of Central and Eastern Madagascar. Missouri Botanical Gardens, Antananarivo.

\section{Biographical sketches}

Dr Thomas Mutschler carried out extensive field research on lemurs in Madagascar, mainly focusing on the Alaotran gentle lemur. His studies cover both applied conservation biology and more academic-oriented research on behavioural ecology. This is also reflected by his affiliations; most of his studies were carried out as a collaboration of the Durrell Wildlife Conservation Trust (Jersey, Channel Islands) and the Anthropological Institute and Museum of University of Zurich (Switzerland). Currently he is doing a post-doctoral study at the Washington University at St Louis (USA) on the taxonomic aspects of vocal communication in wild bamboo lemurs.

Jeannicq Randrianarisoa graduated as an 'Ingenieur' from the Department Eaux et Forets of the Ecole Superieure des Sciences Agronomiques at the University of Atananarivo in 1999, following completion of his 'mémoire de fin d'études' entitled 'Estimation of food intake in wild Alaotran gentle lemurs Hapalemur griseus alaotrensis'. He is currently employed as Head of the Research Department of the Beza Mahafaly project, a conservation project with a special emphasis on research and training in the south of Madagascar.

Anna T.C. Feistner, PhD, is the Head of the Research Department at the Durrell Wildlife Conservation Trust (formerly the Jersey Wildlife Preservation Trust). She is a conservation biologist with broad interests. She runs the research programme at Jersey Zoo and is currently involved in projects in Madagascar, Comores and Sulawesi. She has considerable field experience with primates and has been working with the Alaotran gentle lemur (both wild and captive) for a decade. 